Rakenteiden Mekaniikka (Journal of Structural Mechanics)

Vol. 53, No. 3, 2020, pp. 240-258

http://rakenteidenmekaniikka.journal.fi/index

https://doi.org/10.23998/rm.76502

(C) 2020 The Authors

Open access under license CC BY 4.0

\title{
A plane-stress plasticity model for masonry for the explicit finite element time integration scheme
}

\author{
Oliver Lundqvist ${ }^{1}$ and Michael Chauhan
}

Summary Masonry is a composite material and can be considered anisotropic on a macroscopic scale, i.e. masonry exhibits different properties in different directions, both in the elastic and inelastic range. Like other quasi-brittle materials, masonry exhibits softening and hardening behavior after failure for compression and tension. In this paper a smeared continuum plasticity model of masonry is presented as well as it numerical implementation in an explicit finite element time integration scheme, as such a material model does not exist for a commercial explicit finite element solver. The implementation is done by writing a user-defined material model (VUMAT) as a Fortran subroutine in the commercial software ABAQUS Explicit. The material model is tested both in uniaxial and biaxial loading against similar tests from earlier research. The results show good agreement with earlier research.

Key words: masonry, Rankine, Hill, anisotropic plasticity, super-hyperbolic, cutting plane, VUMAT

Received: 14 November 2018. Accepted: 2 December 2019. Published online: 3 September 2020.

\section{Introduction}

Masonry is a composite construction material which consists of bricks and mortar. The bricks are joined together by embedding the bricks in mortar layers. This jointing method generates a mortar layer pattern, where the horizontal layer is called the bed joint and the vertical mortar layer perpend joint or head joint [6]. Masonry is therefore a material which has distinct directional properties, as the mortar layers act as planes of weaknesses [16]. The directional properties of masonry are dependent on the properties of the brick and mortar as well as on the head and bed joint pattern.

Despite the wide use of masonry around the world and throughout history, the accurate design and analysis of masonry structures remains cumbersome and no general approach seems to exist. Practical design-code based approaches have been implemented,

${ }^{1}$ Corresponding author: oliver.lundqvist@sweco.fi 
and as an example, The European Standard has developed an own Eurocode series, the Eurocode 6, only for the analysis and design of masonry structures [6]. Numerical and simulation-based approaches for masonry structures have been studied and implemented as long as from the 1980s, but no common way for a general modelling approach is set as a "standard". One way of modelling masonry in a finite-element analysis involves a detailed model of the mortar joint with elements or with a simplified connection interface between the bricks. In practice this means building each masonry brick and the mortar joint in detail in the finite element model. This kind of approach may result in tedious manual work for complicated models and structures, not to mention long run times of the analysis. This modelling approach is commonly referred to as "micro-modelling" [11] or "discrete-modelling" [2]. Another numerical method involves a homogenization and smearing of the mortar and brick to a single unified continuum in which the relation between average stresses and strains are established. The smeared approach of masonry can feature anisotropy in both the elastic and inelastic range, and is normally referred to as "macro-modelling" [11] or "smeared-modelling" [2]. This method proves to be practical as normal modelling methods and practices can be used.

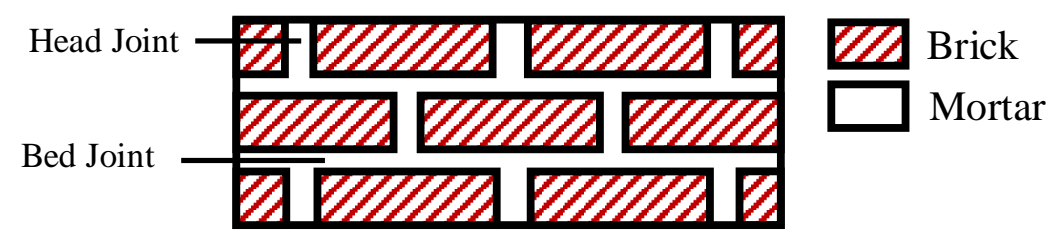

Figure 1. Typical masonry pattern. The figure shows the alignment of the joints.

In recent years, interest in the smeared modelling has occurred as different engineering companies have developed smeared masonry material models for their own business purposes, to use together with commercial software such as ABAQUS and LSDYNA [1, 19]. These developed models are not publicly available and more comprehensive information about these models has not been found. The commercial general-purpose finite-element software DIANA FEA has developed a masonry model together with the Technical University of Delft which can be used within their product [5]. However, the DIANA FEA solver uses an implicit finite element time integration scheme for time history analysis, which might not be applicable for certain types of analyses. As an example, seismic nonlinear time history analysis may require an explicit time integration if the model is complicated and highly nonlinear.

As no commercial finite element software with an explicit time integration scheme and an in-built masonry model exists, this research aims to develop the material model for the explicit integration scheme with a smeared modelling approach. The development is done by first presenting the constitutive behavior of masonry and thereafter implementing the behavior in a general plasticity theory framework. The material is tested by writing a Fortran subroutine which is used within the commercial software ABAQUS Explicit. Even though the developed code will be only compatible with ABAQUS, a similar approach and idea can be used for other finite element explicit solvers (such as the LS-DYNA solver). The Fortran subroutine is tested in single-element uniaxial loading and four-element biaxial loading against reference cases from earlier research. These 
reference cases where chosen as they were the only reference tests that were found from literature for simply modelling the constitutive behavior of the masonry material model.

\section{Plasticity model for masonry}

\section{Failure surface}

The proposed masonry model involves modelling masonry as a smeared quasi-brittle material. Differing from the normal isotropic quasi-brittle material models, the proposed masonry model considers both linear and nonlinear behavior to be different in distinct directions, even for small deformations. The elastic deformations can be considered with classical anisotropic elasticity theory. For the inelastic range, experiments have shown that considering the failure surface in terms of only two principal stresses is not enough as the orientation of the bed joint influences the strength. Page [16] conducted experimental research on the strength of masonry panels in biaxial loading. He tested the biaxial strength of 102 solid clay brick masonry panels by applying different loads and loading rates in the two principle stress directions $\sigma_{I}$ and $\sigma_{I I}$. As a result, Page obtained a failure surface for masonry panels expressed with the two principle stresses $\sigma_{I}$ and $\sigma_{I I}$, and the bed joint orientation angle $\theta$. Ganz and Thülimann [7, 8, 9] conducted similar research where they tested different masonry panels for biaxial loading as well as masonry walls loaded with normal and shear forces. As a result, they obtained tables of different load values and load combinations at failure supporting Page's results.

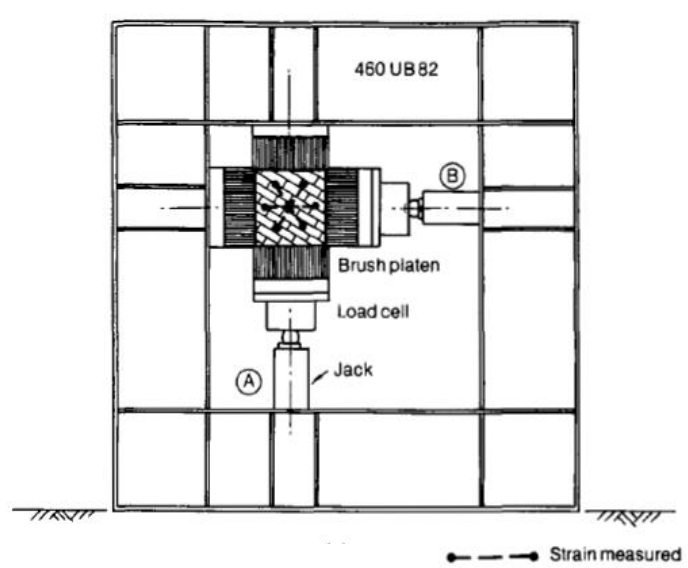

(a)

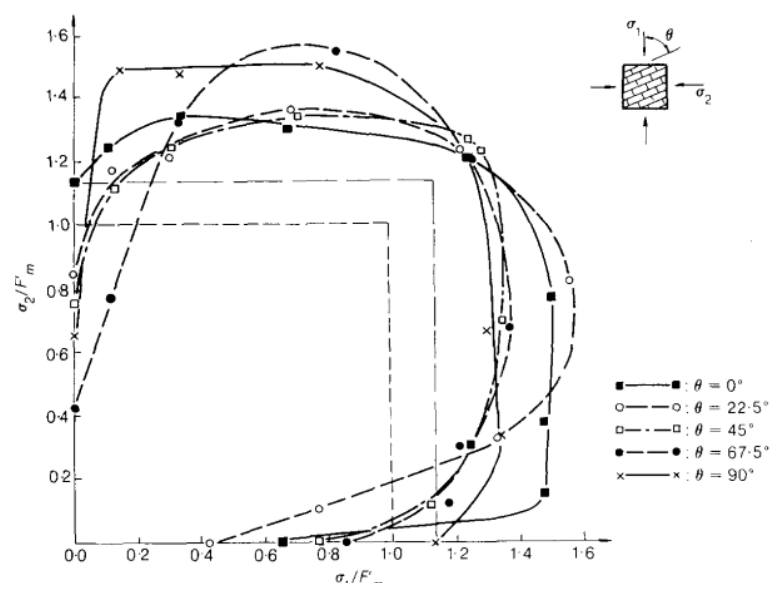

(b)

Figure 2. Failure measurements of masonry panels in biaxial compression. (a) the test setup and (b) the failure surface in $\sigma_{I}-\sigma_{I I}$ plane for different $\boldsymbol{\theta}$ [16].

Another possible representation of the failure surface can be found in terms of spatial directions, $x$ and $y$, where $x$ and $y$ are oriented according to the head and bed joint. In this case, the failure surface is represented by the full plane-stress vector $\left(\sigma_{x}, \sigma_{y}, \tau_{x y}\right)$. For fully determining the yield surface in spatial stresses, seven parameters are needed, which are expressed in 
Table 1. For further details of testing and determining the parameters we refer to [14].

Table 1. Strength parameters for determining the failure surface in spatial stresses

\begin{tabular}{cl}
\hline Parameter & Description \\
\hline$f_{t x 0}$ & initial tensile strength in x-direction \\
$f_{t y 0}$ & initial tensile strength in y-direction \\
$f_{c x 0}$ & peak compressive strength in x-direction \\
$f_{c y 0}$ & peak compressive strength in y-direction \\
$\alpha$ & weight of shear stress contribution to tensile failure \\
$\beta$ & weight of coupling between normal stresses in compressive failure \\
$\gamma$ & weight of shear stress contribution to compressive failure \\
\hline
\end{tabular}

The anisotropic super-hyperbolic Rankine-Hill yield surface

Lourenco et al. $[11,12,13,14]$ presented an analytical yield surface based on a combined yield surface of Rankine and Hill in the spatial stress space $\left(\sigma_{x}, \sigma_{y}, \tau_{x y}\right)$ for masonry. The Rankine failure surface represents the tensile part of the yield surface. Similarly, for compression, a general Hill-type yield criterion is used. This combined multi-surface yield criterion is problematic due to an apex in the tension region which causes numerical instabilities when loading occurs close to the apex. In addition, using these two yield surfaces involves distinguishing which part of the combined yield criteria is violated when failure occurs. Therefore, van der Meer [15] proposed a similar Rankine-Hill yield criterion as an imposed single super-hyperbolic criterion for masonry. This single criterion results in rounding of the apex area and better numerical stability. The yield surface is visualized in Figure 3.

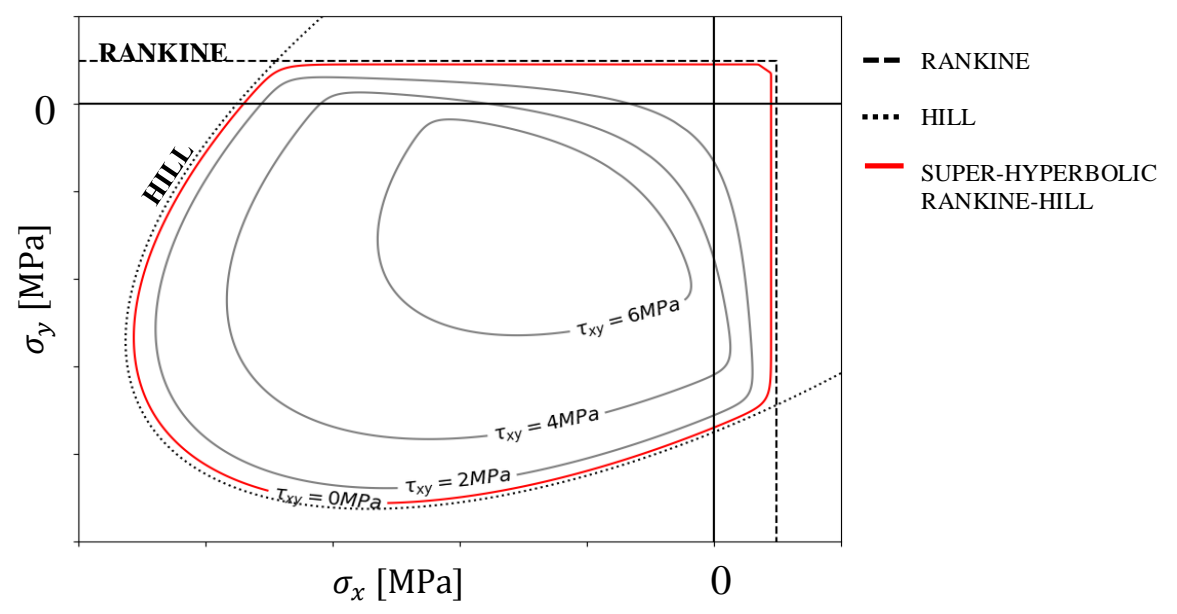

Figure 3. The Super-hyperbolic yield surface in the $\sigma_{x}-\sigma_{y}$ plane and the corresponding Rankine and Hill yield surfaces. in such a way that they satisfy the condition 


$$
\sum_{i} f_{i}^{m}-1 \leq 0
$$

where $m$ is an even integer number, normally taken as 32. Van der Meer utilized equation (1) and split the Rankine and Hill yield criteria in different parts. The Rankine part is defined as

$$
f_{R}=\frac{n_{R}}{d_{R}}
$$

where $n_{R}$ and $d_{R}$ are

$$
\begin{gathered}
n_{R}=\sqrt{\left[\left(\frac{\left(\sigma_{x}-f_{t x}\right)-\left(\sigma_{y}-f_{t y}\right)}{2}\right)^{n}+\mu^{n}\right]+\alpha \tau_{x y}^{2}}, \\
d_{R}=-\frac{\left(\sigma_{x}-f_{t x}\right)-\left(\sigma_{y}-f_{t y}\right)}{2}
\end{gathered}
$$

The variables $f_{t x}$ and $f_{t y}$ are the reduced tensile strength parameters defined in equation (16). The material parameter $\mu$ is defined in equation (17). The power $n$ is normally taken as 16. Similarly, the Hill part is defined for compression as

$$
f_{H}=\frac{n_{H}}{d_{H}}
$$

where $n_{H}$ and $d_{H}$

$$
\begin{gathered}
n_{H}=\sqrt{\beta \sigma_{x} \sigma_{y}+\frac{f_{c x}}{f_{c y}} \sigma_{y}^{2}+\frac{f_{c y}}{f_{c x}} \sigma_{x}^{2}+\gamma \tau_{x y}^{2}}, \\
d_{h}=\sqrt{f_{c x} f_{c y}} .
\end{gathered}
$$

The variables $f_{c x}$ and $f_{c y}$ are the reduced or increased compressive strength parameters defined in equations (18) to (20). Combing and inserting equations (2) to (7) into (1) with some manipulation yields

$$
f_{R H}=\frac{1}{d_{H}}\left(n_{R}^{m} d_{H}^{m}+n_{H}^{m} d_{R}^{m}\right)^{1 / m}-d_{R} \leq 0,
$$

which is the super-hyperbolic Rankine-Hill yield surface. The yield surface is presented in Figure 3. 


\section{Hardening and softening}

Usually, formulations of hardening and softening for quasi-brittle isotropic material consider different inelastic criteria for tension and compression. As masonry is also considered to be a quasi-brittle material, it includes similar hardening and softening features. For tension, the post-failure behavior consists of an exponential softening in both distinct directions. On the other hand, the compression has an initial hardening phase, after which a softening phase follows. The hardening and softening for compression are also distinct in different directions. Figure 4 shows typical inelastic characteristics for tension and compression.

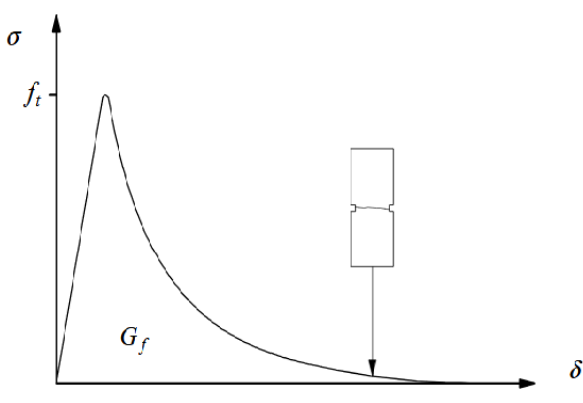

(a)

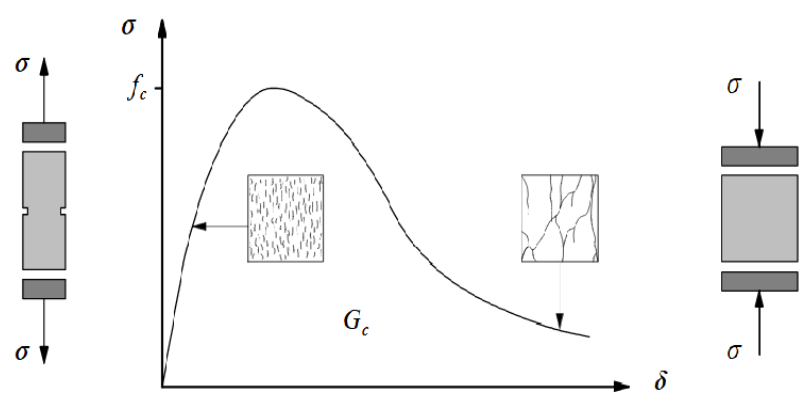

(b)

Figure 4. (a) Uniaxial tension with softening and (b) uniaxial compression with initial hardening and a softening branch [11].

For the prescribed model, the development of plastic flow is assumed to be associated, i.e. the yield function serves as the plastic potential. Thereby the plastic strain is defined by the plastic multiplier $\lambda$ and yield surface $f_{R H}$ as

$$
\varepsilon_{p l}=\lambda \frac{\partial f_{R H}}{\partial \sigma},
$$

The evolution of hardening and softening is described with a hardening law. The state of the evolution is controlled by the hardening variable $\kappa$. The hardening law is defined as

$$
\boldsymbol{\kappa}=\lambda \boldsymbol{h},
$$

where $\boldsymbol{h}$ is the hardening modulus. The hardening or softening is distinct for both tension and compression and noted as $\kappa_{t}$ and $\kappa_{c}$, respectively.

To implement compression hardening or softening and tension softening for the given yield surface, the plastic strain must be separable into parts belonging to Rankine surface and Hill surface, hence

$$
\varepsilon_{p l}=\lambda \frac{\partial f_{R H}}{\partial \sigma}=\lambda\left(\frac{\partial f_{R}}{\partial \sigma}+\frac{\partial f_{H}}{\partial \sigma}\right)
$$


The hardening modulus $\boldsymbol{h}$ is thereby distinguishable in a Rankine (tension) and Hill (compression) part. Van der Meer [15] gives the Rankine softening moduli $h_{R}$ as

$$
h_{R}=\sqrt{\frac{1}{2}\left(\frac{\partial f_{R}}{\partial \sigma}\right)^{T} \boldsymbol{Q} \frac{\partial f_{R}}{\partial \sigma}}+\frac{1}{2} \boldsymbol{\pi}^{T} \frac{\partial f_{R}}{\partial \sigma}
$$

where the matrix $\boldsymbol{Q}$ and vector $\boldsymbol{\pi}$ are defined as

$$
\begin{aligned}
& \boldsymbol{Q}=\left[\begin{array}{ccc}
\frac{1}{2} & -\frac{1}{2} & 0 \\
-\frac{1}{2} & \frac{1}{2} & 0 \\
0 & 0 & \frac{1}{2}
\end{array}\right], \\
& \boldsymbol{\pi}=\left(\begin{array}{lll}
1 & 1 & 0
\end{array}\right)^{T} .
\end{aligned}
$$

The Hill hardening and softening moduli $h_{H}$ is given as

$$
h_{H}=\frac{1}{d_{H}} \boldsymbol{\sigma}^{T} \frac{\partial f_{H}}{\partial \sigma} .
$$

The actual tension softening law can be expressed as a decaying exponential function of the tensile strength $f_{t x}$ and $f_{t y}$. Van der Meer [15] gives one possibility as

$$
f_{t i}\left(\kappa_{t}\right)=\frac{5}{2} \mu+\left(f_{t i 0}-\frac{5}{2} \mu\right) \exp \left(-\frac{h f_{t i 0}}{G_{t i}}\right), \quad i=x, y
$$

where $\kappa_{t}$ is the tension softening variable, $G_{t i}$ is the fracture energy and $h$ is the characteristic element length (typically taken as the square root of the area). The idea of utilizing fracture energy together with the characteristic element length is to obtain an energy release independent of the mesh size and hence avoid mesh sensitivity [11]. The parameter $\mu$ controls the residual strength in equation (16) and improves the numerical stability. It is defined as

$$
\mu=\frac{2 \mu_{t o l}}{5} \min \left(f_{t x 0}, f_{t y 0}\right)
$$

where $\mu_{t o l}$ is a small arbitrary value, normally between 0.01 and 0.1 , for controlling the residual strength.

For the compression, which considers both hardening and softening, the law for the post-failure behavior is more complex. The increase and decrease of the compression strengths $f_{c x}$ and $f_{c y}$ are given as the partly defined function 


$$
\begin{gathered}
f_{c i}\left(\kappa_{c}\right)=f_{c i 0}-b_{i}+b_{i} \sqrt{1-\left(\frac{\kappa_{c}-\kappa_{p}}{a_{i}}\right)^{2}}, \quad \kappa_{c}<\kappa_{p} \\
f_{c i}\left(\kappa_{c}\right)=f_{c i 0}-\frac{1}{2} f_{c i 0}\left(\frac{\kappa_{c}-\kappa_{p}}{\kappa_{m i}-\kappa_{p}}\right)^{2}, \quad \kappa_{p} \leq \kappa_{c}<\kappa_{m i} \\
f_{c i}\left(\kappa_{c}\right)=\frac{1}{10} f_{c i 0}+\frac{2}{5} f_{c i 0} \exp \left(m_{i} \frac{\kappa_{c}-\kappa_{m i}}{\frac{2}{5} f_{c i 0}}\right), \quad \kappa_{m i} \leq \kappa_{c} \\
i=x, y,
\end{gathered}
$$

where $\kappa_{c}$ is the hardening variable, $f_{c x 0}$ and $f_{c y 0}$ are the peak compression strengths and $\kappa_{p}$ the value of the hardening variable at peak compression. The additional parameters $a_{i}, b_{i}, \kappa_{m i}$ and $m_{i}$ are only for shorter equation formulations of equations (18) to (20) and defined as

$$
\begin{gathered}
m_{i}=\frac{-f_{c i 0}}{\kappa_{m i}-\kappa_{p}}, \\
k_{m i}=\frac{75}{67} \frac{G_{c i}}{h f_{c i 0}}+\kappa_{p}, \\
a_{i}=\sqrt{\frac{\kappa_{p}\left(-\frac{1}{2} f_{c i 0}+H_{0} \kappa_{p}\right)^{2}}{H_{0}\left(-\frac{1}{2} f_{c i 0}+H_{0} \kappa_{p}\right)}}, \\
b_{i}=\frac{-\frac{1}{2} f_{c i 0}}{\sqrt{\frac{\left(-\frac{1}{2} f_{c i 0}\right)^{2}}{\left(-\frac{1}{2} f_{c i 0}+H_{0} \kappa_{p}\right)^{2}}-1}} .
\end{gathered}
$$

The parameter $H_{0}$ in equation (23) is an arbitrary large value that determines the initial slope of the hardening. The compression hardening and softening described in equations (18) to (24) is visualized in Figure 5.

For fully determining the hardening and softening of the material model, the parameters in Table 2 need to be defined together with the tensile and compressive strengths $f_{t x 0}, f_{t y 0}, f_{c x 0}$ and $f_{c y 0}$. 


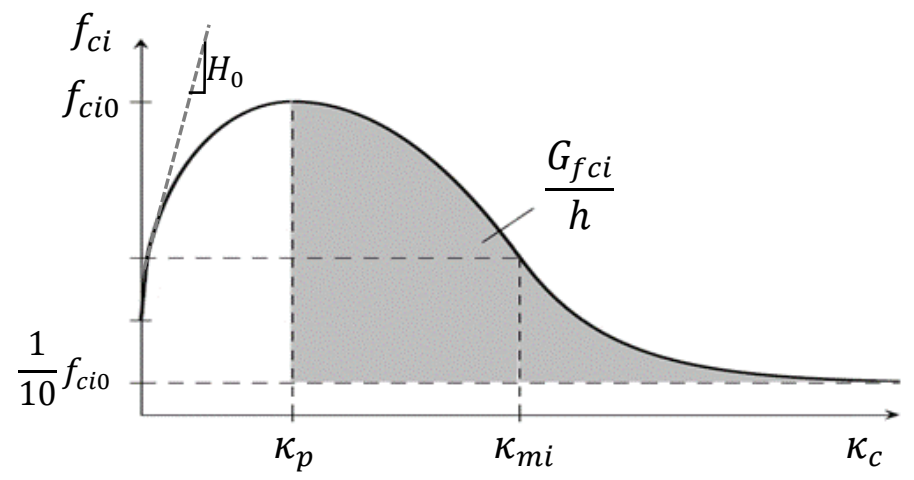

Figure 5. Illustration of equations (18) to (24) for the compression hardening and softening [11].

Table 2. Material parameters for determining hardening and softening

\begin{tabular}{cl}
\hline Parameter & \multicolumn{1}{c}{ Description } \\
\hline$G_{t x}$ & tensile fracture energy in $x$-direction \\
$G_{t y}$ & tensile fracture energy in $y$-direction \\
$G_{c x}$ & compressive fracture energy in $x$-direction \\
$G_{c y}$ & compressive fracture energy in $y$-direction \\
$\kappa_{p}$ & peak strain for peak compressive stress \\
$\mu_{t o l}$ & small numerical value for residual strength in tension softening \\
$H_{0}$ & large numerical value for controlling the initial slope in compression \\
& hardening \\
\hline
\end{tabular}

\section{Finite element implementation}

\section{Explicit finite elements}

Given the dynamic explicit finite element discretization as

$$
\boldsymbol{M} \frac{\partial^{2} \boldsymbol{u}}{\partial t^{2}}=\boldsymbol{F}-\boldsymbol{I}
$$

it follows to find the displacement $\boldsymbol{u}$ for time $t$, given the internal force vector $\boldsymbol{I}$, external force vector $\boldsymbol{F}$ and the lumped mass matrix $\boldsymbol{M}$ [3]. To solve for the displacements $\boldsymbol{u}$, equation (25) is further discretized in the time domain for numerical time integration. One possible discretization is the central difference rule which is utilized by ABAQUS [4]. This leads to a straight-forward method as

$$
\ddot{u}_{n}=M^{-1}\left(F_{n}-I_{n}\right)
$$




$$
\begin{gathered}
\dot{\boldsymbol{u}}_{n+\frac{1}{2}}=\dot{\boldsymbol{u}}_{\boldsymbol{n}-\frac{1}{2}}+\frac{\Delta t_{n+1}+\Delta t_{n}}{2} \ddot{\boldsymbol{u}}_{\boldsymbol{n}} \\
\boldsymbol{u}_{n+1}=\boldsymbol{u}_{\boldsymbol{n}}+\Delta t_{n+1} \dot{\boldsymbol{u}}_{n+\frac{1}{2}}
\end{gathered}
$$

The subscripts in equations (26) to (28) refer to discretization of the time $t$. The double dot sign in the previous equations correspond to acceleration (second derivative of displacement with respect to time) and the single dot to velocity (first derivative of the displacement with respect to time). The time increment $\Delta t$ is calculated during the analysis. To be able to use equations (26) to (28), the initial velocity states need to be defined and are given by the user.

The internal force vector $\boldsymbol{I}$ in equation (25) is constructed by evaluating the stresses at the integration points. The internal force vector is taken as

$$
I=\int B^{T} \sigma d V
$$

where $\boldsymbol{B}$ is the Jacobian matrix of the element shape functions. As the stresses are a function of the strains and the central difference integration evaluates the displacement field for the next time increment, it is possible to evaluate the stresses at the beginning of the time increment and hence construct the internal force vector at the beginning of each time step. In commercial finite element software, this opens the possibility to write userdefined material models. Usually in ABAQUS or LS-DYNA, these material models are written as Fortran subroutines and included in the analysis $[4,10]$. For ABAQUS Explicit, this kind of subroutine is called a "VUMAT" and for LS-DYNA "MAT_USER_DEFINED_MATERIAL_MODELS". For the VUMAT, ABAQUS calls the subroutine at each time increment to determine stresses at each integration point. The subroutine receives the strain increment $\Delta \boldsymbol{\varepsilon}$, old stresses $\sigma_{n}$ and the old state variables $\boldsymbol{\varepsilon}_{\boldsymbol{p l}, \boldsymbol{n}}$ and $\boldsymbol{\kappa}_{\boldsymbol{n}}$ as an input, and the subroutine determines the new stresses $\boldsymbol{\sigma}_{\boldsymbol{n}+\boldsymbol{1}}$ and new state variables $\boldsymbol{\varepsilon}_{\boldsymbol{p l}, \boldsymbol{n + 1}}$ and $\boldsymbol{\kappa}_{\boldsymbol{n}+\boldsymbol{1}}$ for the new time $t_{n+1}$. The internal force vector $\boldsymbol{I}$ is constructed by the solver itself. It is also possible to calculate the internal energies and internal inelastic energies within the subroutine.

\section{Implemented material model}

The failure model for masonry is implemented as an ABAQUS VUMAT subroutine. The material model assumes the strain increment to be given by the analysis at each time step. The task remains to determine the stresses at each time step. The material model is divided conventionally into an elastic and plastic part where the plastic part is initialized if yield criterion (8) is violated. The material model can be summarized with the constitutive equations:

$$
\text { STRESS-STRAIN RELATIONSHIP: } \quad \boldsymbol{\sigma}=\boldsymbol{C}\left(\boldsymbol{\varepsilon}-\boldsymbol{\varepsilon}_{\boldsymbol{p l}}\right) \text {, }
$$




$$
\begin{aligned}
& \text { FLOW RULE: } \boldsymbol{\varepsilon}_{\boldsymbol{p} \boldsymbol{l}}=\lambda \frac{\partial f_{R H}}{\partial \boldsymbol{\sigma}}, \\
& \text { HARDENING LAW: } \quad \boldsymbol{\kappa}=\lambda \boldsymbol{h}, \\
& \text { YIELD CRITERION: } f_{R H}(\boldsymbol{\sigma}, \boldsymbol{\kappa})=\frac{1}{d_{H}}\left(n_{R}^{m} d_{H}^{m}+n_{H}^{m} d_{R}^{m}\right)^{1 / m}-d_{R},
\end{aligned}
$$

subjected to the Kuhn-Tucker loading or unloading conditions

$$
\begin{gathered}
\lambda \geq 0, \\
f_{R H}(\boldsymbol{\sigma}, \boldsymbol{\kappa}) \leq 0, \\
\lambda f_{R H}(\boldsymbol{\sigma}, \boldsymbol{\kappa})=0,
\end{gathered}
$$

and consistency condition

$$
\lambda f_{R H}^{\cdot}(\boldsymbol{\sigma}, \boldsymbol{\kappa})=0
$$

The implementation of the material model for the constitutive equations thereby require to determine the new state $\left(\boldsymbol{\varepsilon}_{\boldsymbol{p l}, \boldsymbol{n}+\mathbf{1}}, \boldsymbol{\kappa}_{\boldsymbol{n}+\mathbf{1}}, \boldsymbol{\sigma}_{\boldsymbol{n}+\mathbf{1}}\right)$, given the previous state $\left(\boldsymbol{\varepsilon}_{\boldsymbol{p l}, \boldsymbol{n}}, \boldsymbol{\kappa}_{\boldsymbol{n}}, \boldsymbol{\sigma}_{\boldsymbol{n}}\right)$ and the strain increment $\Delta \boldsymbol{\varepsilon}$, so that the equations (30) to (37) hold. One approach for solving this problem is to utilize an elastic predictor and plastic corrector integration scheme. This integration scheme assumes the first increment to be completely elastic. If the elastic predictor violates the yield criterion, the stresses are corrected with a plastic corrector to the feasible stress space, i.e. projected back to the yield surface. This approach transforms the constitutive equations (30) to (37) into a set of nonlinear algebraic equations that need to be solved. Solving this kind of problem is normally referred to as return mapping and involves usually an iterative scheme for obtaining a solution. For simple elastic-plastic models, analytical and elegant solutions may be found. Nevertheless, one possible method for integration of the constitutive equation is the cutting-plane algorithm which is used for the return mapping in this model. For further details and derivation of the cutting-plane algorithm, we refer to [17, 18].

Given the strain increment for a given time $t_{n+1}$, the elastic predictor can be calculated with the stress-strain relationship (30) where it is assumed that the elasticity matrix $\boldsymbol{C}$ is orthotropic and defined as

$$
\boldsymbol{C}=\left[\begin{array}{ccc}
E_{x} & \frac{v E_{x}}{1-v^{2}} & 0 \\
\frac{v E_{y}}{1-v^{2}} & E_{y} & 0 \\
0 & 0 & G_{x y}
\end{array}\right]
$$


The elastic predictor is then

$$
\sigma_{n+1}=\sigma_{n}+C \Delta \varepsilon
$$

The material parameters $E_{x}, E_{y}, v$ and $G_{x y}$ refer to the Young's moduli in different directions, Poisson's ratio and the shear modulus, respectively.

If the calculated elastic predictor stress violates the yield criterion (33), the stresses are returned to the yield surface and the feasible stress space by the corrector step. This is done by increasing the plastic strain and hence lowering the stresses. Thereby the equations need to be solved for $\lambda$. The cutting-plane algorithm is a straightforward method for solving $\lambda$, and is geometrically illustrated in Figure 6 . The cutting-plane algorithm is an explicit scheme and has a quadratic convergence rate [17].

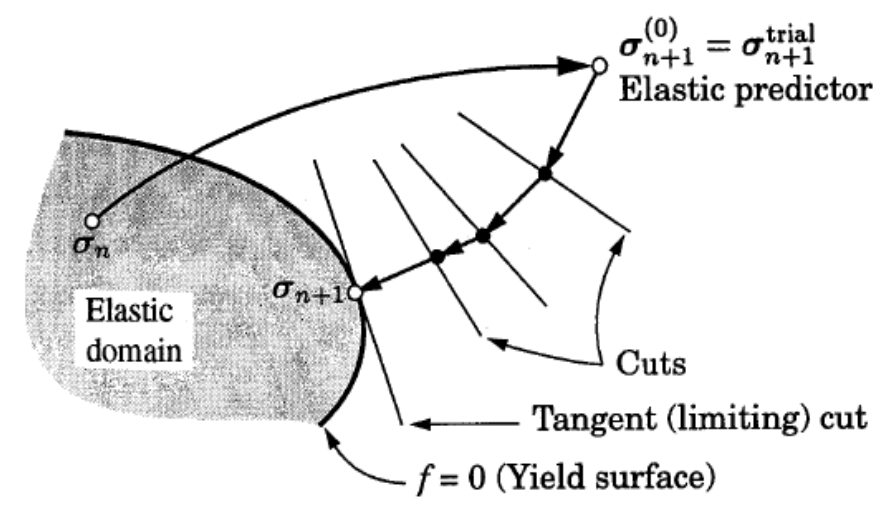

Figure 6. Illustration of the cutting-plane algorithm [17].

The cutting-plane algorithm is implemented for the prescribed material model and the procedure is summarized below.

\section{INPUT:}

$\Delta \boldsymbol{\varepsilon}=$ total strain increment

$\sigma_{\boldsymbol{n}}=$ stress at previous time step

$\boldsymbol{\kappa}_{\boldsymbol{n}}=$ hardening-softening variable at previous time step

$\boldsymbol{\varepsilon}_{\boldsymbol{p l}, \boldsymbol{n}}=$ plastic strain at previous time step

\section{ADDITIONAL VARIABLES:}

$f_{R H}=$ yield surface

$\lambda=$ plastic increment

$\Delta \varepsilon_{p l}=$ plastic strain increment

\section{OUTPUT:}

$\sigma_{n+1}=$ updated stress

$\boldsymbol{\varepsilon}_{\boldsymbol{p l}, \boldsymbol{n + 1}}=$ updated plastic strain

$\boldsymbol{\kappa}_{n+1}=$ updated hardening-softening variable 
1. Initialize:

$$
\Delta \boldsymbol{\varepsilon}_{\boldsymbol{p l}}^{(0)}=\mathbf{0}, \quad \boldsymbol{\kappa}_{\boldsymbol{n}+\mathbf{1}}^{(k)}=\boldsymbol{\kappa}_{\boldsymbol{n}}, \quad \lambda^{(0)}=0, \quad k=0
$$

2. Calculate elastic stress:

$$
\boldsymbol{\sigma}_{n+1}^{(k)}=\sigma_{n}+C\left(\Delta \varepsilon-\Delta \varepsilon_{p l}^{(k)}\right)
$$

Calculate hardening moduli

$$
\boldsymbol{h}_{\boldsymbol{n}+\mathbf{1}}^{(k)}=h\left(\boldsymbol{\sigma}_{\boldsymbol{n}+\mathbf{1}}^{(k)}, \boldsymbol{\kappa}_{\boldsymbol{n}+\mathbf{1}}^{(k)}\right)
$$

Calculate yield surface:

$$
f_{R H}^{(k)}=f\left(\boldsymbol{\sigma}_{\boldsymbol{n}+\mathbf{1}}^{(k)}, \boldsymbol{\kappa}_{\boldsymbol{n}+\mathbf{1}}^{(k)}\right)
$$

3. Calculate plastic increment:

$$
\lambda^{(k)}=\frac{f_{R H}^{(k)}}{\left(\partial f_{R H}^{(k)} / \partial \boldsymbol{\sigma}_{\boldsymbol{n}+\mathbf{1}}^{(\boldsymbol{k})}\right)^{T} \boldsymbol{C}\left(\partial f_{R H}^{(k)} / \partial \boldsymbol{\sigma}_{\boldsymbol{n}+\mathbf{1}}^{(\boldsymbol{k})}\right)+\left(\partial f_{R H}^{(k)} / \partial \boldsymbol{\kappa}_{\boldsymbol{n}+\mathbf{1}}^{(\boldsymbol{k})}\right)^{T} \boldsymbol{h}_{\boldsymbol{n}+\mathbf{1}}^{(\boldsymbol{k})}}
$$

4. Update variables:

$$
\begin{gathered}
\Delta \boldsymbol{\varepsilon}_{\boldsymbol{p l}}^{(k+1)}=\Delta \boldsymbol{\varepsilon}_{\boldsymbol{p l}}^{(k)}+\lambda^{(k)}\left(\partial f_{R H}^{(k)} / \partial \boldsymbol{\sigma}_{\boldsymbol{n}+\mathbf{1}}^{(k)}\right) \\
\boldsymbol{\kappa}_{\boldsymbol{n}+\mathbf{1}}^{(k+1)}=\boldsymbol{\kappa}_{\boldsymbol{n}}^{(k)}+\lambda^{(k)} \boldsymbol{h}_{\boldsymbol{n}+\mathbf{1}}^{(k)}
\end{gathered}
$$

5. Go to 2 and set $k \leftarrow k+1$ and repeat $\mathrm{N}$ times.

6. After $\mathrm{N}$ iterations update the plastic strains:

$$
\varepsilon_{p l, n+1}=\varepsilon_{p l, n}+\Delta \varepsilon_{p l}^{(N)}
$$

Note that in step 2, the yield criterion is not checked and the iteration loop is not stopped and exited if $\boldsymbol{f}_{\boldsymbol{R} \boldsymbol{H}}^{(\boldsymbol{k})} \leq \mathbf{0}$, instead we do a fixed amount of iterations for robustness. 


\section{Reference tests of the material model}

For evaluating the performance of the developed material model, three small reference cases were selected for comparison:

1) a single-element test for uniaxial compression and tension in both $x$ and $y$ directions, where the reference case was done by Lourenco [11],

2) a single-element test for uniaxial compression and tension in both $x$ and $y$ directions, where the reference case was done by van der Meer [15],

3) a four-element test for biaxial compression and tension, where the reference case was done by van der Meer [15].

The material model used by van der Meer was the same as the presented material model. However, van der Meer's material model or any masonry model in a commercial finite element software has not been implemented in an explicit finite element integration scheme and hence we seek to apply van der Meer's model in an explicit finite element integration scheme. Both Lourenco's and van der Meer's material models were used and developed for an implicit finite element time integration scheme. Also, the material model used by Lourenco is slightly different as it was developed earlier, and van der Meer made some improvements to the model.

The reference models were built in ABAQUS Explicit. A four-node shell element, $\mathrm{S} 4 \mathrm{R}$, was used in the analysis, with three integration points through the thickness. The size of the element depended on the reference case and is shown in Table 3 . The loading was applied by giving the element edge a prescribed displacement in the direction of interest. The load was ramped up with ABAQUS's smooth step function. As the reference tests were done with an implicit solver as a static case, a high simulation time was chosen, i.e. $30 s$ to avoid any dynamical effects. In addition, the time step was kept small for stability. The used time step varied between $2-10 \mu$ s. The analysis was run with double precision, for both the analysis and the packager. For the four-element biaxial load case, a load rate of 1:2 in $x$ and $y$ direction was used.

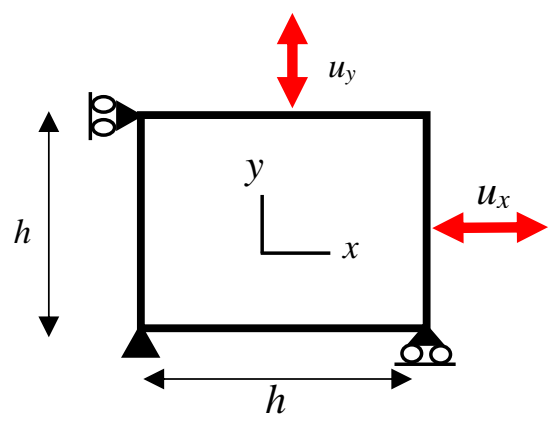

(a)

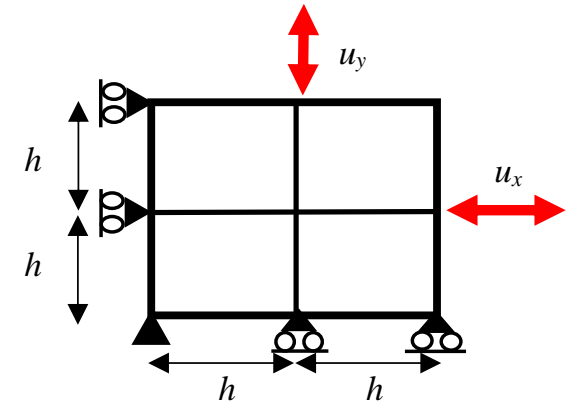

(b)

Figure 7. The setup for the ABAQUS models: (a) the single-element model and (b) the fourelement model.

The material model was implemented as a Fortran VUMAT subroutine with the parameters given in Table 4. The material values for the reference case of van der Meer and Lourenco, were taken directly from [15] and [11]. The meaning of the material 
parameters $f_{t x 0}, f_{t y 0}, f_{c x}, f_{c y}, \alpha, \beta$ and $\gamma$ are summarized in Table 2 and the parameters $G_{t x} G_{t y}, G_{c x}, G_{c y}, \kappa_{p}, \mu_{t o l}$ and $H_{0}$ are summarized in Table 3 . The derivation of the beforementioned parameters from tests are described in [14].

Table 3. Element size of reference cases.

\begin{tabular}{cccc}
\hline Reference case & 1-E Lourenco & 1-E van der Meer & 4-E van der Meer \\
\hline$h[\mathrm{~mm}]$ & 100 & 424 & 300 \\
\hline
\end{tabular}

Table 4. Used material parameters.

\begin{tabular}{ccc}
\hline Parameter & Lourenco & Van der Meer \\
\hline$E_{x}[\mathrm{MPa}]$ & 10000 & 14000 \\
$E_{y}[\mathrm{MPa}]$ & 5000 & 14000 \\
$G_{x y}[\mathrm{MPa}]$ & 3000 & 5833.33 \\
$v[-]$ & 0.2 & 0.2 \\
$f_{t x 0}[\mathrm{MPa}]$ & 1.0 & 0.2 \\
$f_{t y 0}[\mathrm{MPa}]$ & 0.5 & 1.0 \\
$G_{t x}[\mathrm{~N} / \mathrm{mm}]$ & 0.02 & 0.0255 \\
$G_{t y}[\mathrm{~N} / \mathrm{mm}]$ & 0.006 & 0.127 \\
$f_{c x 0}[\mathrm{MPa}]$ & 10 & 10 \\
$f_{c y 0}[\mathrm{MPa}]$ & 5 & 20 \\
$G_{c x}[\mathrm{~N} / \mathrm{mm}]$ & 5 & 46.7 \\
$G_{c y}[\mathrm{~N} / \mathrm{mm}]$ & 1.6 & 93.3 \\
$\alpha[-]$ & 1 & 1 \\
$\beta[-]$ & -1 & -1 \\
$\gamma[-]$ & 3 & 3 \\
$H_{0}[\mathrm{MPa}]$ & 100000 & 100000 \\
$\kappa_{p}[-]$ & 0.0005 & 0.002 \\
$\mu_{t o l}[-]$ & 0.01 & 0.1 \\
\hline & &
\end{tabular}

\section{Results}

\section{Single-element uniaxial load reference tests}

The results for the uniaxial single element test cases are shown in Figure 8 and Figure 9. The red color indicates the developed and presented material model and is noted as "VUMAT" in the legend. 


\section{Four-element biaxial load reference tests}

The results for the biaxial four element test cases are shown in Figure 11 and Figure 10. The red color indicates the developed and presented material model and is noted as "VUMAT" in the legend.

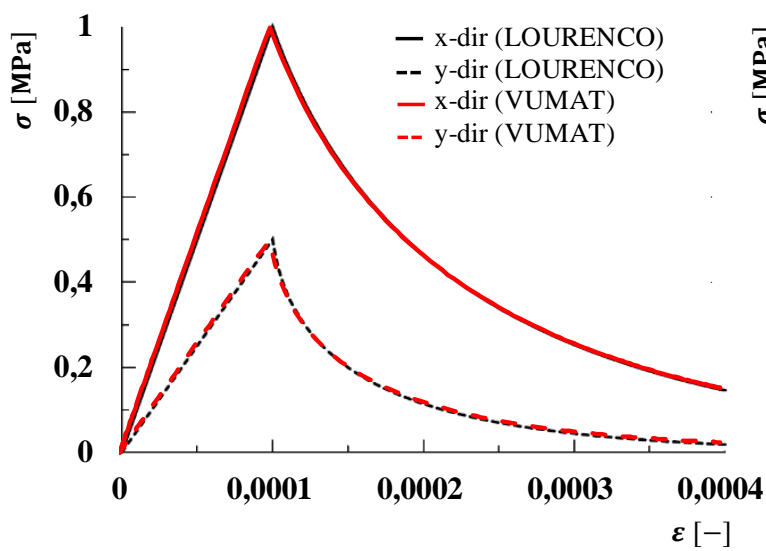

(a)

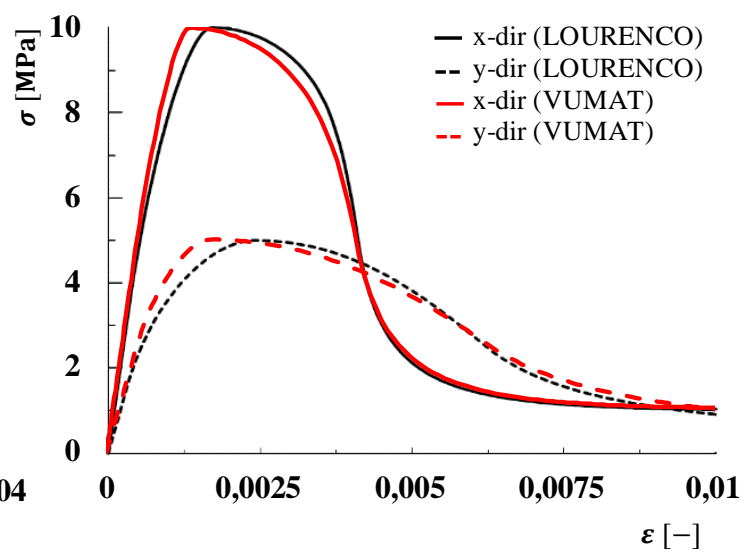

(b)

Figure 8. Comparison of results between the developed material model for (a) tension tests and (b) compression tests. Comparison results from Lourenco [11].

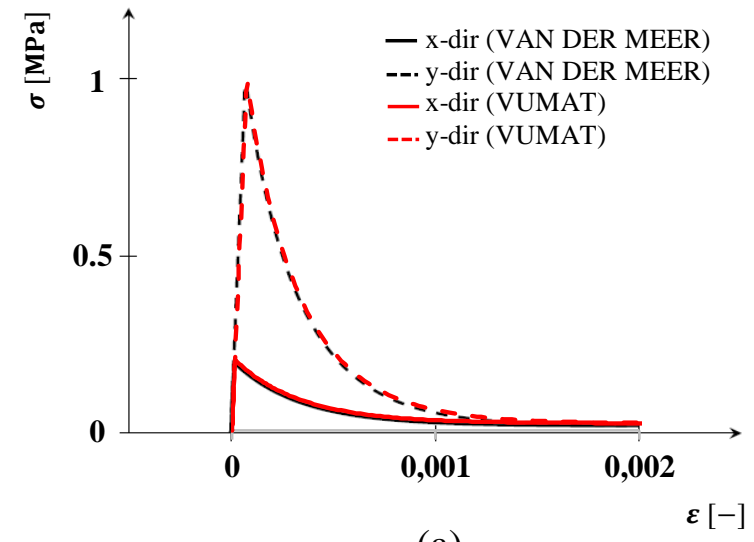

(a)

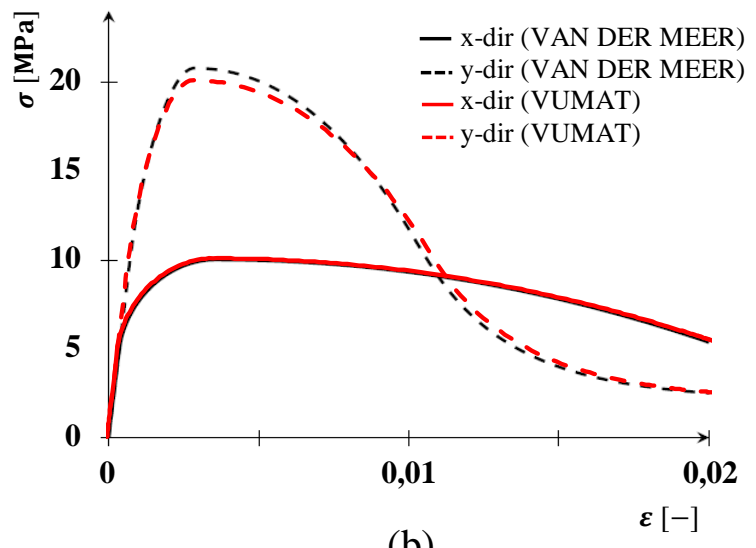

(b)

Figure 9. Comparison of results between the developed material model for (a) tension tests and (b) compression tests. Comparison results from van der Meer [15]. 


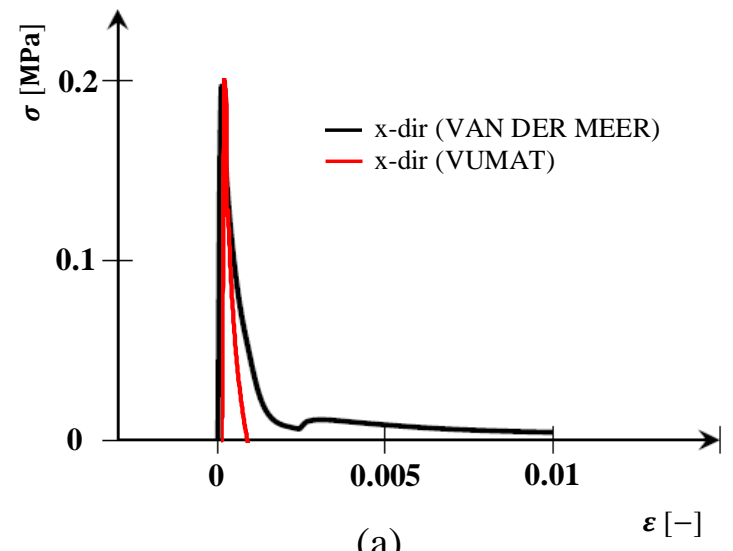

(a)

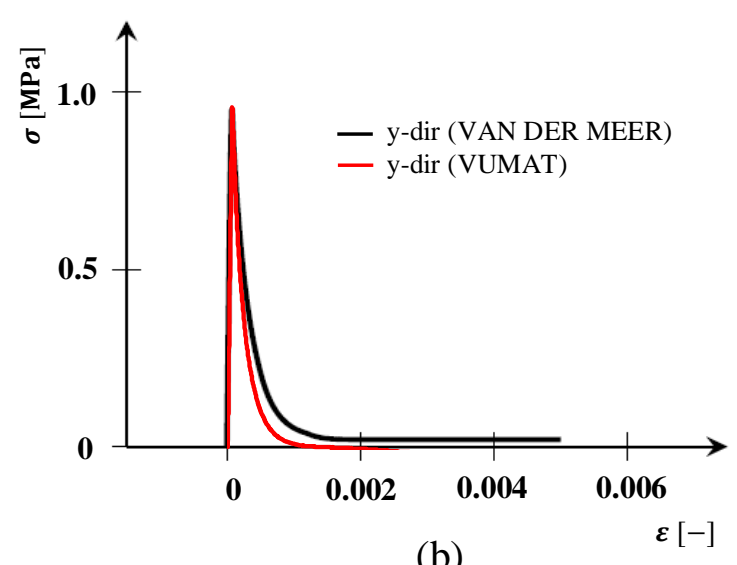

(b)

Figure 11. Comparison of results from the biaxial tension loading test for (a) $x$-direction and (b) $y$ direction. Comparison results from van der Meer [15].

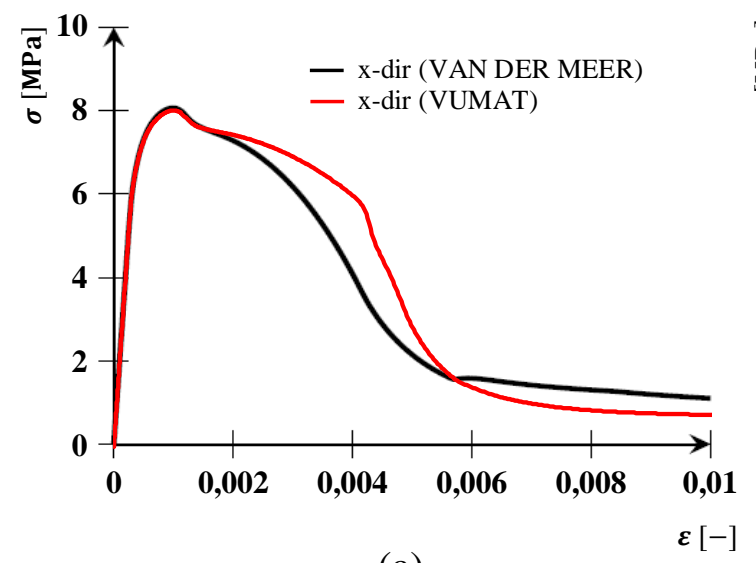

(a)

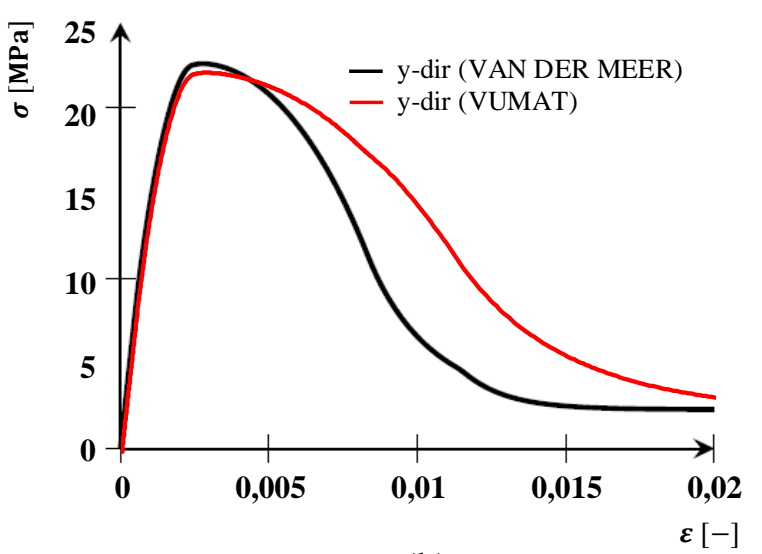

(b)

Figure 10. Comparison of results from the biaxial compression loading test for (a) $\mathrm{x}$-direction and (b) y-direction. Comparison results from van der Meer [15].

\section{Discussion}

For the uniaxial single-element tests, good agreement between the developed material model and the reference cases were found. The stress-strain behavior of the developed material model followed the same results obtained by van der Meer [15]. A minor difference for compression between the developed material model and the results from Lourenco [11] can be seen. The deviation is explained by the difference in the analytical expressions for compression hardening, where Lourenco uses a slightly different analytical formula for determining the compression stress. 
The four-element tests show agreement with the results obtained by van der Meer [15]. However, for the tension test case the developed material model showed a sharper drop in strength than the reference case. One explanation for this is the use of an explicit solver where static equilibrium is not checked nor necessary. The elements showed an uneven stress distribution when failure occurred, which might be caused by the lack of convergence and equilibrium checks. For compression, the developed material model showed more capacity in the softening phase. The reason for this might also be the uneven stress distribution which occurred. Nonetheless, the obtained results are still comparable to the reference case done by van der Meer.

Running the analysis showed sensitivity to the time step. When using larger time increments (size of $100 \mu \mathrm{s}$ ) non-convergence occurred in the return mapping. Decreasing the time increments by a factor of 10 solved the non-convergence issue. For future cases, this issue might be problematic where large strains and failure can occur rapidly. For the cutting-plane algorithm, it is known that using larger increments might cause problems and incorrect results. For future work we propose to investigate the stability of the return mapping by using implicit return mappings such as the closest-point-projection (fully implicit backward Euler) for the integration of the constitutive equations (30) to (37).

Material models utilizing a strain softening approach are known to be mesh sensitive. In this research, mesh sensitivity was not an issue as the model was tested on a few elements and compared to reference cases of literature [11,15] with the same element size. Nonetheless, the softening phase of the presented model is handled with a fracture energy-based regularization using a characteristic element length (square root of element area), which is a common practice in the field to overcome mesh sensitivity.

The comparison was a study of the constitutive behavior of the material model and its implementation in an explicit finite element integration scheme. The model was not tested on meshes with larger number of elements due to the lack of simple reference cases with large meshes. For future work, we propose to investigate the behavior, sensitivity and stability of the material model for different meshes and mesh sizes.

The presented and tested material model could be used together with commercial finite element software with an explicit time integration scheme for estimating the failure behavior of masonry structures. For the implicit finite element solver one commercially available software exists with an in-built masonry material model. Nonetheless, the implicit time integration is unpractical when solving highly dynamic and nonlinear problems. Therefore, the development of a masonry material model is highly motivated as in-built masonry material models do not exist at the current time for commercial finite element explicit solvers.

\section{References}

[1] A. Aukselis. Homogeneous orthotropic masonry research, development and implementation for explicit analysis. Master's Thesis, Delft University of Technology. 2017.

[2] T. T. Bakeer. Collapse analysis of masonry structures under earthquake actions. $\mathrm{PhD}$, Technische Universität Dresden. 2009. 
[3] T. Belytschko, W. K. Liu, B. Moran, K. Elkhodary. Nonlinear finite elements for continua and structures. $2^{\text {nd }}$ edition. John Wiley \& Sons Ltd. 2014

[4] Dassault Systemes Simulia Corp. Abaqus theory guide. 2016.

[5] Diana Fea Bv. User's manual-release 10.2. 2017.

[6] EN1996-1-1 Eurocode 6. Design of masonry structures - Part-1-1: General rules for reinforced and unreinforced masonry structures. CEN, 2005.

[7] H. R. Ganz, B. Thürlimann. Versuche über die Festigkeit von zweiachsig beanspruchtem Mauerwerk. Working Paper, Swiss Federal Institute of Technology in Zurich. 1982.

DOI: https://doi.org/10.3929/ethz-a-000248407

[8] H. R. Ganz, B. Thürlimann. Versuche an Mauerwerksscheiben unter Normalkraft und Querkraft . Working Paper, Swiss Federal Institute of Technology in Zurich. 1984.

DOI: https://doi.org/10.3929/ethz-a-000328538

[9] H. R. Ganz. Mauerwerksscheiben unter normalkraft und schub. $\mathrm{PhD}$, Swiss Federal Institute of Technology in Zurich. 1985. DOI: https://doi.org/10.3929/ethz-a-000360363

[10] Livermore Software Technology Corp. LS-DYNA keyword user's manual volume II material models. R10.0. 2017.

[11] P. B. Lourenco. Computational strategies for masonry structures. $\mathrm{PhD}$, Delft University of Technology. 1996.

[12] P. B. Lourenco, R. de Borst, J. G. Rots. A plane stress softening plasticity model for orthotropic materials. Int. J. Numer. Meth. Engng., 40, 4033-4057, 1997.

[13] P. B. Lourenco, J. G. Rots. Multisurface interface model for analysis of masonry structures. Journal of Engineering Mechanics 123(7) 660-668, 1997.

[14] P. B. Lourenco, J. G. Rots, J. Blaauwendraad. Continuum model for masonry: parameter estimation and validation. Journal of Structural Engineering. 124(6), 642-652, 1998.

[15] L. J. van der Meer. Unbonded post-tensioned shear walls of calcium silicate element masonry. PhD, Eindhoven University of Technology. 2013.

[16] A. Page. The biaxial compressive strength of brick masonry. Proceedings of the Institution of Civil Engineers, 71(3) part 2, 893-906, 1981. DOI: https://doi.org/10.1680/iicep.1981.1825

[17] J. C Simo, T. J. R Hughes. Computational Inelasticity. Springer-Verlag, New York. 2000.

[18] E. A. de Souza Neto, D. Peric, D. R. J. Owen. Computational methods for plasticity theory and applications. John Wiley \& Sons Ltd. 2008.

[19] E. C. M. van Wijnbergen. Exploration and analysis of low-cost seismic retrofit measures to improve box-action for traditional brick masonry houses in Nepal. Master's Thesis, Delft University of Technology. 2016.

Oliver Lundqvist, Michael Chauhan

Sweco Rakennetekniikka Oy

Ilmalanportti 2, 00240 Helsinki

oliver.lundqvist@sweco.fi, michael.chauhan@sweco.fi 